\title{
A Note on Product Measures and Representations of the Canonical Commutation Relations
}

\author{
Ole A. Nielsen \\ Queen's University, Kingston, Canada
}

Received March 3, 1971

\begin{abstract}
There is a well-known theorem which states that a non-zero $\sigma$-finite left quasi-invariant measure on a $\sigma$-compact locally compact group $G$ must be equivalent to left Haar measure. It is shown in this paper that there is a natural generalization of this fact to the case in which the group $G$ is replaced by a product space, one factor of which is a group. With the aid of this generalization, an easy proof of the following fact, due to $\mathrm{H}$. Araki, is given: the representations of the canonical commutation relations constructed in the usual measure-theoretic manner are ray continuous.
\end{abstract}

Almost invariably, the most desirable measure on a product space is a product measure. However, in doing integration theory on a product space, one is sometimes confronted with a measure which is a priori not even equivalent to a product measure. It is therefore of some interest to find conditions under which a measure on a product space must be equivalent to a product measure. Theorem 1 below gives such a condition which is, roughly speaking, that one of the factors be a group and that the measure in question be quasi-invariant under the action of the group on the product space. Araki proved Theorem 1 for the special case of a Euclidean group [1; Lemma 5.2]. His proof relies on the ray continuity of the representations of the canonical commutation relations (CCRs) constructed in the usual measure-theoretic manner (see [1; Section 1]). It was pointed out by Araki that the converse is also true, i.e., that ray continuity could be deduced from Theorem 1 . In fact, the ray continuity is an easy consequence of Theorem 2, which itself depends on Theorem 1.

Suppose that $G$ is a $\sigma$-compact locally compact group, that $\mathscr{A}$ is its $\sigma$-algebra of Borel sets (i.e., the $\sigma$-algebra generated by the open sets), and that $\lambda$ is a left-invariant Haar measure on $(G, \mathscr{A})$. Suppose further that $\mathscr{B}$ is a $\sigma$-algebra of subsets of a non-empty set $Z$. Let $\mathscr{A} \times \mathscr{B}$ be the product $\sigma$-algebra on $G \times Z$. Measurability of subsets of or functions defined on $G$ [resp., $Z, G \times Z]$ will always be taken with respect to $\mathscr{A}$ [resp., $\mathscr{B}$, $\mathscr{A} \times \mathscr{B}]$. Setting $x(y, \zeta)=(x y, \zeta)$ for all $x, y \in G$ and all $\zeta \in Z$ defines a left action of $G$ on $G \times Z$. The characteristic function of a subset $S$ of $G \times Z$ will be denoted by $1_{s}$.

Theorem 1. Suppose that $v$ and $\mu$ are $\sigma$-finite measures on $(G \times Z$, $\mathscr{A} \times \mathscr{B})$ and $(Z, \mathscr{B})$, resp. Then $v$ is equivalent to $\lambda \times \mu$ if and only if 

and

(i) for each $x \in G$ and $S \in \mathscr{A} \times \mathscr{B}, v(S)=0$ if and only if $v(x S)=0$,

(ii) for each $B \in \mathscr{B}, \mu(B)=0$ if and only if $v(G \times B)=0$.

Proof. With no loss of generality, $v$ and $\mu$ may both be assumed to be finite. Notice that $\lambda$ is $\sigma$-finite since $G$ is, by hypothesis, $\sigma$-compact. If $v$ is equivalent to $\lambda \times \mu$, then clearly (ii) must hold, and by Fubini's theorem, so must (i). Conversely, suppose that (i) and (ii) are satisfied.

Fix, for the moment, a measurable subset $S$ of $G \times Z$. Then

$$
S[x]=\{\zeta \in Z:(x, \zeta) \in S\}
$$

is measurable for each $x$ in $G([2 ;$ p. 141, Theorem A]), and the function $(x, y, \zeta) \rightarrow 1_{s}\left(x^{-1} y, \zeta\right)$ is measurable on $G \times G \times Z$ with respect to the product $\sigma$-algebra. If $\Delta$ is the modular function of $G$, then

$$
\begin{aligned}
\int_{G \times Z} \int_{G} 1_{S}\left(x^{-1} y, \zeta\right) & d \lambda(x) d v(y, \zeta) \\
& =\int_{G \times Z} \int_{G} \Delta(x) 1_{S}(x, \zeta) d \lambda(x) d v(y, \zeta) \\
& =\int_{G} \int_{G \times Z} \Delta(x) 1_{G \times S[x]}(y, \zeta) d v(y, \zeta) d \lambda(x) \\
& =\int_{G} \Delta(x) v(G \times S[x]) d \lambda(x)
\end{aligned}
$$

by an application of Tonelli's theorem ([2; p. 147, Theorem B]). On the other hand,

$$
\int_{G} \int_{G \times Z} 1_{S}\left(x^{-1} y, \zeta\right) d v(y, \zeta) d \lambda(x)=\int_{G} v(x S) d \lambda(x) .
$$

So by a second application of Tonelli's theorem

$$
\int_{G} v(x S) d \lambda(x)=\int_{G} \Delta(x) v(G \times S[x]) d \lambda(x) .
$$

Since the modular function is everywhere positive, the last equation means that $v(x S)=0$ for $\lambda$-a.a. $x \in G$ if and only if $v(G \times S[x])=0$ for $\lambda$-a.a. $x \in G$. Now by (i), $v(x S)=0$ for $\lambda$-a.a. $x \in G$ is equivalent to $v(S)=0$. The collection of all those measurable subsets $S$ of $G \times Z$ for which $x \rightarrow \mu(S[x])$, is a measurable function on $G$ is a monotone class which includes the measurable rectangles, and is therefore all of $\mathscr{A} \times \mathscr{B}[2$; p. 27, Theorem A]. From condition (ii), $v(G \times S[x])=0$ for $\lambda$-a.a. $x \in G$ if and only if $\mu(S[x])=0$ for $\lambda$-a.a. $x \in G$, which, by Fubini's theorem, is equivalent to $\lambda \times \mu(S)=0$.

Theorem 1 is a generalization of the fact that any left quasi-invariant measure on $G$ is equivalent to $\lambda$. In fact, the proofs of this result and of Theorem 1 are similar (see [5; Lemma 3.3]). Say $H$ is a closed subgroup of $G$. In view of the fact that the left coset space $G / H$ carries a unique left quasi-invariant measure class ([6; Lemma 1.3$])$, it is hardly surprising 
that Theorem 1 remains valid when the factor $G$ is replaced by $G / H$. This extension of the theorem will now be sketched.

Let $H$ be a closed subgroup of $G$, and let $\lambda_{H}$ be its left-invariant Haar measure. Let $p: G \rightarrow G / H$ be the natural projection of $G$ onto the left coset space $G / H$. Let $\mathscr{A}_{H}$ denote the largest $\sigma$-algebra of subsets of $G / H$ making $p$ measurable, and let $\lambda^{*}$ be the measure on $\left(G / H, \mathscr{A}_{H}\right)$ defined by $p$ and some finite measure $\lambda^{\prime}$ on $(G, \mathscr{A})$ equivalent to $\lambda$. Now suppose that $\mu$ and $v$ are finite measures on $(Z, \mathscr{B})$ and $\left(G / H \times Z, \mathscr{A}_{H} \times \mathscr{B}\right)$, resp., which satisfy and

(iii) for each $x \in G$ and $S \in \mathscr{A}_{H} \times \mathscr{B}, v(S)=0$ if and only if $v(x S)=0$,

(iv) for each $B \in \mathscr{B}, \mu(B)=0$ if and only if $v(G / H \times B)=0$.

(In (iii), $x S$ means the image of $S$ under the natural left action of $x$ on $G / H$.) A modification of the argument employed by Mackey in $[6$; Lemma 1.3] gives a $\sigma$-finite measure $\sigma$ on $(G \times Z, \mathscr{A} \times \mathscr{B})$ which satisfies (i), (ii), and $\sigma\left(p^{-1}(E)\right)=\lambda_{H}(H) v(E)$ for each $E$ in $\mathscr{A}_{H} \times \mathscr{B}$. Then $\sigma$ is equivalent to $\lambda^{\prime} \times \mu$, and it readily follows that $v$ must be equivalent to $\lambda^{*} \times \mu$. This proves the non-trivial part of the following result.

Corollary. With the above notation, $v$ and $\lambda^{*} \times \mu$ are equivalent if and only if $v$ and $\mu$ satisfy (iii) and (iv).

Suppose now that $v$ is a finite measure on $(G \times Z, \mathscr{A} \times \mathscr{B})$ which satisfies (i). Let $v_{t}(S)=v(t S)$ for all $t$ in $G$ and all sets $S$ in $\mathscr{A} \times \mathscr{B}$. For each $t \in G$ and each $\psi \in L^{2}(G \times Z, \mathscr{A} \times \mathscr{B}, v)=L^{2}(v)$, put

$$
[V(t) \psi](x, \zeta)=\left[\frac{d v_{t}}{d v}(x, \zeta)\right]^{1 / 2} \psi(t x, \zeta)
$$

for all $(x, \zeta) \in G \times Z$. It is readily verified that $t \rightarrow V(t)$ is a unitary representation of $G$ on $L^{2}(v)$. It will be shown below that this representation is even strongly continuous.

If $\mu$ denotes the finite measure $B \rightarrow v(G \times B)$ on $(Z, \mathscr{B})$, then $v$ and $\lambda \times \mu$ must be equivalent (Theorem 1). Let $f$ be the Radon-Nikodym derivative of $v$ with respect to $\lambda \times \mu$. Then $f$ can be chosen to satisfy $0<f(x, \zeta)<\infty$ for all points $(x, \zeta)$ in $G \times Z$. For any function $\psi$ on $G \times Z$, set $\psi_{t}(x, \zeta)=\psi(t x, \zeta)$ for all $t, x \in G$ and $\zeta \in Z$. An easy calculation shows that

$$
v_{t}(S)=\int_{S} f_{t} d \lambda \times \mu
$$

for each $t \in G$ and all $S \in \mathscr{A} \times \mathscr{B}$. Thus $\frac{d v_{t}}{d \lambda \times \mu}=f_{t} v$-a.e., and so $\frac{d v_{t}}{d v}=\frac{f_{t}}{f}$ $v$-a.e. $[2 ;$ p. 133, Theorem A].

Suppose that $\psi_{1}$ and $\psi_{2}$ are two functions in $L^{2}(v)$. Since the weak and strong topologies coincide on the unitary operators, it is sufficient to 
show that the inner product of $V(t) \psi_{1}$ and $\psi_{2}$ in $L^{2}(v)$ depends continuously on $t$. This inner product is

$$
\begin{aligned}
\int_{G \times Z}\left[V(t) \psi_{1}\right] \bar{\psi}_{2} d v & =\int_{G \times Z}\left(f_{t} / f\right)^{1 / 2} \psi_{1 t} \bar{\psi}_{2} f d \lambda \times \mu \\
& =\int_{G \times Z}\left(f^{1 / 2} \psi_{1}\right)_{t}\left(f^{1 / 2} \bar{\psi}_{2}\right) d \lambda \times \mu .
\end{aligned}
$$

The functions $\left(f^{1 / 2} \psi_{1}\right)_{t}$ and $f^{1 / 2} \psi_{2}$ are in $L^{2}(G \times Z, \mathscr{A} \times \mathscr{B}, \lambda \times \mu)$ $=L^{2}(\lambda \times \mu)$, and the last integral is their inner product in this Hilbert space. Consequently, it is enough to show that $t \rightarrow \psi_{t}$ is strongly continuous from $G$ to $L^{2}(\lambda \times \mu)$ for each $\psi$ in $L^{2}(\lambda \times \mu)$. Now this is certainly the case if $\psi(x, \zeta)=\psi_{1}(x) \psi_{2}(\zeta)$ for some $\psi_{1}$ in $L^{2}(G, \mathscr{A}, \lambda)$ and $\psi_{2}$ in $L^{2}(Z, \mathscr{B}, \mu)$ [4; Theorem $30 C]$. Since the finite linear combinations of such functions are strongly dense in $L^{2}(\lambda \times \mu)$, the proof of the following theorem is complete.

Theorem 2. If $v$ is a finite measure on $(G \times Z, \mathscr{A} \times \mathscr{B})$, then the equation

$$
[V(t) \psi](x, \zeta)=\left[\frac{d v_{t}}{d v}(x, \zeta)\right]^{1 / 2} \psi(t x, \zeta)
$$

defines a strongly continuous unitary representation $V$ of $G$ on $L^{2}(G \times Z, \mathscr{A} \times \mathscr{B}, v)$.

Araki proved the special case of Theorem 2 in which $G$ is the real line [1; Lemma 2.3]. The ray continuity of the representations of the CCRs constructed in the usual measure-theoretic manner follows easily from this special case of Theorem 2 (see [1; Theorem 2.4 and its proof]).

In conclusion, it should be noted that G. Hegerfeldt has also given a short proof of the ray continuity [3; Corollary 3.5].

\section{References}

1. Araki,H.: On representations of the canonical commutation relations. Commun. math. Phys., 20, 9-25 (1971).

2. Halmos, P.R.: Measure theory. New York: Van Nostrand 1950.

3. Hegerfeldt, G.C.: On canonical commutation relations and infinite-dimensional measures, to appear.

4. Loomis, L. H.: An introduction to abstract harmonic analysis. New York: Van Nostrand 1953.

5. Mackey, G. W.: On a theorem of Stone and von Neumann. Duke Math. J. 16, 313-326 (1949).

6. - Induced representations of locally compact groups. I, Ann. Math. 55, 101-139 (1952).

O. A. Nielsen

Department of Mathematics

Jefferey Hall

Queen's University

Kingston, Ontario, Canada 